PROCEEDINGS OF THE

AMERICAN MATHEMATICAL SOCIETY

Volume 125, Number 8, August 1997, Pages 2463-2469

S 0002-9939(97)04071-9

\title{
ON THE DOUBLE CURVES OF LEAST AREA TORI
}

\author{
MAX NEUMANN-COTO
}

(Communicated by Ronald Stern)

\begin{abstract}
The double curves of least area immersions of the torus into closed, orientable, irreducible 3-manifolds are simple in the torus. A related result for other least area surfaces is given.
\end{abstract}

If $f: F \rightarrow M$ is a general position immersion of a surface into a 3-dimensional manifold, then the set of points where $f(F)$ fails to be an embedding consists of a finite collection of double curves and triple points. The preimages of these curves in $F$, also known as double curves, may not be simple or disjoint (they meet at the preimages of the triple points). A double curve in $f(F)$ is simple in $M$ if and only if the corresponding double curves in $F$ (2 if $F$ and $M$ are orientable) are simple and disjoint. Figure 1 shows the double curves of 3 homotopic immersions of the torus into some 3-manifold and illustrates how much they can change with the homotopy.

Of special interest are immersions which are incompressible $\left(\pi_{1}\right.$-injective $)$ and have least area in its free homotopy class for some Riemannian metric on the manifold. In this case one can hope that the singularities of the immersion are minimal and are related to the topology of $M$. The immersions in Figure 1 are incompressible, and the last 2 have least area for some Riemannian metrics.

Freedman, Hass and Scott [2] proved that if $M$ is orientable and irreducible (i.e. every sphere embedded in $M$ bounds a 3-ball), and $F$ is orientable and not $S^{2}$, then the double curves of any least area incompressible immersion $f: F \rightarrow M$ are essential in $F$. In general however, these double curves are not disjoint nor simple, and even their number may vary depending on the metric given to the manifold. In the case of least area tori much more can be said. In his proof of the Torus Theorem [1] Casson has shown that if $f: T^{2} \rightarrow M$ is a least area incompressible immersion of a torus into a closed, orientable and irreducible manifold, then:

(i) All the double curves of $f$ represent powers of the same element of $\pi_{1}\left(T^{2}\right)$, unless $M$ is covered by $S^{1} \times S^{1} \times S^{1}$.

(ii) The double curves of $f$ represent primitive elements of $\pi_{1}\left(T^{2}\right)$, so they are homotopic to simple curves in $T^{2}$.

In this paper a similar approach is used to show that the double curves of a least area immersion $f: T^{2} \rightarrow M$ are in fact simple in $T^{2}$, and that if $F$ is any orientable surface and $f: F \rightarrow M$ has the so-called 1-line property, then the double curves of $f$ represent primitive elements of $\pi_{1}(F)$. Finally, examples of least area tori in which every double curve intersects each other are given.

Although the image of a least area incompressible surface $f: F \rightarrow M$ may fail to be in general position, or even be self-transverse, the image of a least area

Received by the editors October 11, 1995.

1991 Mathematics Subject Classification. Primary 57M60, 57R45.

Key words and phrases. 3-manifolds, immersions, minimal surfaces. 

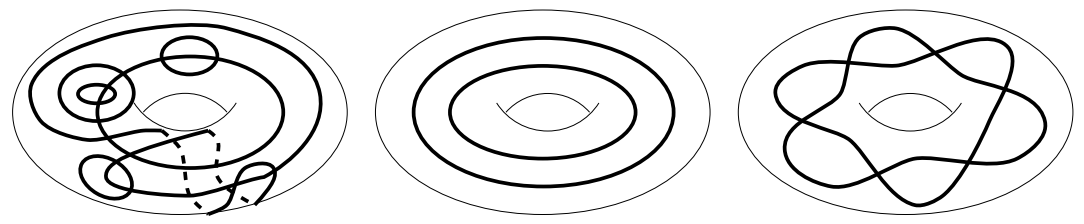

Figure 1

incompressible torus is always a self-transverse torus or Klein bottle [2]. This doesn't imply that the image is in general position: there may be curves or arcs where more than two sheets of the image meet, but one can regard these multiple intersections as many simple intersections that coincide. We want to show that the preimages of these curves of transverse intersection of $f\left(T^{2}\right)$ are simple curves in $T^{2}$.

Freedman, Hass and Scott showed in [2] that if $f: F \rightarrow M$ is a least area incompressible immersion of an orientable surface into an orientable and irreducible 3 -manifold, then the preimage of $f(F)$ in the universal covering $\tilde{M}$ of $M$ consists of embedded (topological) planes. Moreover, if the immersion is self-transverse, then the intersection between planes consists of topological lines, and no closed curves. So the double curves are essential in $F$. An immersion is said to have the 1-line property if its preimage in $\tilde{M}$ consists of planes which intersect transversally in at most 1 line. They showed that the image of a least area torus always has the 1-line property, but this may fail for other least area surfaces. They proved that if $f: F \rightarrow M$ has the 1-line property, then the number and free homotopy classes of the double curves in $F$ are the same for all least area maps homotopic to $f$ independently of the Riemannian metric on $M$.

Let $P$ be one of the planes in $\tilde{M}$ that cover $f\left(T^{2}\right)$. We can identify $\pi_{1}(M)$ with the group of covering translations of $\tilde{M}$, and identify $f_{*} \pi_{1}\left(T^{2}\right)$ with the subgroup $S_{P}$ of covering translations that stabilize $P$ and preserve its orientation. The preimage in $P$ of each double curve in $f\left(T^{2}\right)$ consists of two families of lines of intersection of $P$ with other planes, one for each of the two preimages of the double curve in $T^{2}$. Each family is an orbit of the action of $S_{P}$ on the lines of intersection in $P$. As $P / S_{P}^{+} \simeq T^{2}$, the lines in each family are parallel in $P$, in the sense that they are all stabilized by the same covering translations. To prove that a double curve in $T^{2}$ is simple, we must show that the lines in its family are disjoint. The next theorem shows that the planes that contain them are disjoint.

Theorem 1. Let $\tilde{M}$ be the universal covering of a closed, orientable, irreducible 3-manifold $M$, and let $P$ and $Q$ be two planes in $\tilde{M}$ covering a torus with the 1-line property in $M$. If $P \cap Q \neq \emptyset$, then any two translates of $P$ by the action of $S_{Q}$ are disjoint or equal.

Proof. We want to show that if $\alpha \in S_{Q}$ does not stabilize $P$, then $\alpha P \cap P=\emptyset$. Let $l$ denote the line of intersection of $P$ and $Q$, and let $l_{i}$ denote the line $P \cap \alpha^{i} P$ whenever $\alpha^{i} P$ intersects $P$ transversally. Then $\alpha^{n} P$ intersects $Q$ at $\alpha^{n} l$, and if $\alpha^{n} P$ intersects $\alpha^{m} P$, it does so at the line $\alpha^{n} l_{m-n}$. In principle some of these lines may be the same. In the rest of the proof by planes we mean $Q, P$ and its translates, and by lines the lines of intersection between them. Let's call two lines parallel if there is a covering translation that stabilizes both lines. 

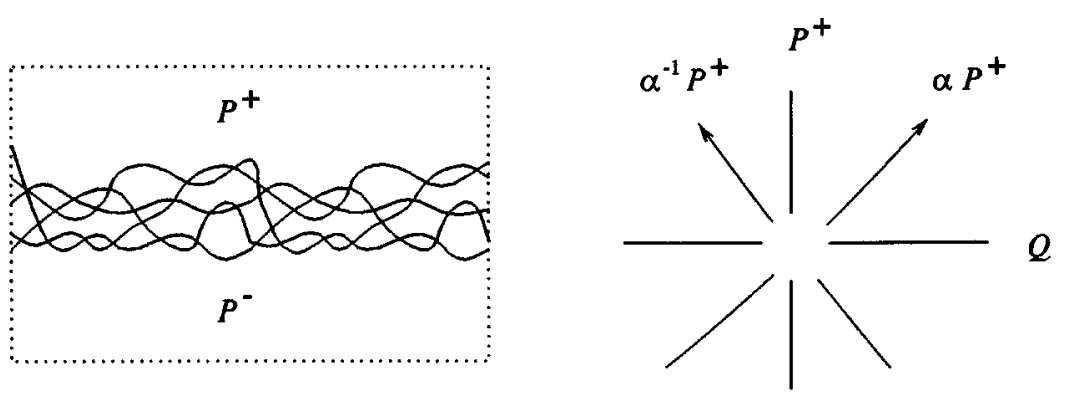

FiguRe 2

Lemma 1.1. The lines $l, l_{i}$, and all their translates by powers of $\alpha$ are parallel in $\tilde{M}$.

Proof. The group of covering translations that stabilize $l$ is infinite cyclic, and as $S_{P}$ and $S_{Q}$ have infinite cyclic subgroups that stabilize $l, S_{P} \cap S_{Q}$ is an infinite cyclic group. If $\lambda$ is its generator, then $\alpha$ and $\lambda$ commute because $S_{Q} \simeq \mathbb{Z}+\mathbb{Z}$. Therefore $\lambda\left(\alpha^{i} P\right)=\alpha^{i} \lambda P=\alpha^{i} P$, so $\lambda$ stabilizes all the planes and therefore stabilizes the intersections between them.

Let $\tilde{M} / \lambda$ denote the quotient of $\tilde{M}$ by the action of $\langle\lambda\rangle$. The lines project to closed curves in $\tilde{M} / \lambda$, and the planes project to embedded annuli (and not Moebius bands) because $\lambda$ preserves the orientation of $Q$. Orient all the lines in the same direction (i.e., so that they project to the same generator of $H_{1}(\tilde{M} / \lambda)=\mathbb{Z}$ ). As the action of $\alpha$ preserves the orientations of the lines in $P$, it preserves the orientations of all the lines.

The following is a convenient way to define orientations: the points in a plane outside the neighborhood of a line determine two sides of the plane, and parallel lines determine the same sides (see Figure 2a). One can also think of the sides of $P$ as the preimages of the 2 ends of the annulus $P / \lambda$. The orientation of the lines and the choice of the positive side $P^{+}$of $P$ determine an orientation for $P$, and if $P^{\prime}$ intersects $P$, then the orientation of the intersection line and the choice of $P^{+}$and $P^{\prime+}$ determine an orientation for $\tilde{M}$, denoted by $\left(P^{+}, P^{\prime+}\right) .\left(P^{+}, P^{\prime+}\right)$ and $\left(P^{+}, P^{\prime \prime+}\right)$ determine the same orientation if and only if ${P^{\prime+}}^{+}$and $P^{\prime \prime}+$ lie on the same component of $\tilde{M}-P$. Choose $P^{+}$and use the action of $\alpha$ to define the positive sides of its translates, and observe that $\alpha$ preserves the sides of $Q$.

Lemma 1.2. If $P$ intersects $\alpha P$, then $\alpha P^{+}$and $\alpha^{-1} P^{+}$lie on different components of $\tilde{M}-P$.

Proof. We need to show that $\left(P^{+}, \alpha P^{+}\right)$and $\left(P^{+}, \alpha^{-1} P^{+}\right)$determine opposite orientations on $M$. Clearly $\left(P^{+}, \alpha^{-1} P^{+}\right)$and $\left(\alpha^{-1} P^{+}, P^{+}\right)$determine opposite orientations, and $\alpha$ maps $\left(\alpha^{-1} P^{+}, P^{+}\right)$to $\left(P^{+}, \alpha P^{+}\right)$preserving orientation.

Lemma 1.3. If $P$ intersects $\alpha P$, then $P$ intersects $\alpha^{2} P$.

Proof. Clearly $P$ intersects $\alpha P$ if and only if $P$ intersects $\alpha^{-1} P$. Figure 2b suggests that then $\alpha P$ must intersect $\alpha^{-1} P$, which is what we need to prove. Consider the orientations defined by $\left(Q^{+}, \alpha P^{+}\right),\left(Q^{+}, \alpha^{-1} P^{+}\right),\left(P^{+}, \alpha P^{+}\right)$and $\left(P^{+}, \alpha^{-1} P^{+}\right)$. Since $\alpha \in S_{Q}$ the first two are equal, and by Lemma 1.2 the last two are opposite. 


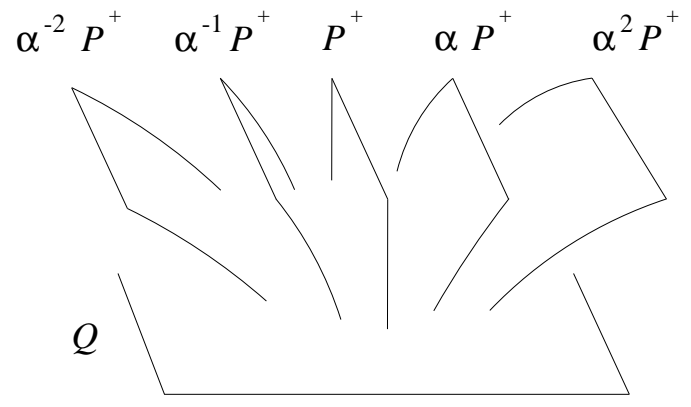

Figure 3

If $\alpha P$ and $\alpha^{-1} P$ were disjoint, they would cobound a region $X$ in $\tilde{M}$, and the sides of $P$ and $Q$ would lie on $\tilde{M}-X$. Now if $Q^{+}$and $P^{+}$were on the same component of $\tilde{M}-X$, then $\left(Q^{+}, \alpha P^{+}\right)$and $\left(P^{+}, \alpha P^{+}\right)$would be equal, and $\left(Q^{+}, \alpha^{-1} P^{+}\right)$and $\left(P^{+}, \alpha^{-1} P^{+}\right)$would be equal. And if $Q^{+}$and $P^{+}$were on different components of $\tilde{M}-X$, then $\left(Q^{+}, \alpha P^{+}\right)$and $\left(P^{+}, \alpha P^{+}\right)$would be opposite, and $\left(Q^{+}, \alpha^{-1} P^{+}\right)$and $\left(P^{+}, \alpha^{-1} P^{+}\right)$would be opposite.

Lemma 1.4. For $n>0, \alpha P^{+}$and $\alpha^{n} P^{+}$lie on the same component of $\tilde{M}-P$.

Proof. We want to show that the translates of $P^{+}$are placed as in Figure 3. Call the component of $\tilde{M}-Q$ that contains $P^{+}$above and the other component below, and call the component of $\tilde{M}-\alpha^{i} P$ that contains $Q^{+}$the right of $\alpha^{i} P$ and the other the left. Assume inductively that $\alpha P^{+}$and $\alpha^{n} P^{+}$are on the same component of $\tilde{M}-P$, say on the right of $P$. Then $\alpha^{n+1} P \neq P$, otherwise $\alpha^{n+1} P^{+}=P^{+}$, and so $\alpha^{n} P^{+}=\alpha^{-1} P^{+}$, but $\alpha P^{+}$and $\alpha^{-1} P^{+}$are on different components of $\tilde{M}-P$.

We need to show that $\alpha^{n+1} P^{+}$is not on the left of $P$. As $\alpha$ maps points above $Q$ to points above $Q$ and it sends $Q^{+}$to $Q^{+}$, then $\alpha^{n+1} P^{+}$is above $Q$ and on the right of $\alpha^{n} P$. Let $X$ be the set of points in $\tilde{M}$ above $Q$, on the right of $\alpha^{n} P$ and on the left of $P . X$ is invariant under $\lambda$. We claim that $X / \lambda$ is compact, and so $\alpha^{n+1} P^{+} / \lambda$ (an end of the annulus $\alpha^{n+1} P / \lambda$ ) can't be contained in it. The boundary of $X$ is contained in $P \cup \alpha^{n} P \cup Q$, and it doesn't contain $P^{+}, P^{-}, \alpha^{n} P^{+}, \alpha^{n} P^{-}, Q^{+}$or $Q^{-}$ $\left(Q^{+}\right.$is on the right of $P$ and $Q^{-}$is on the left of $\alpha^{n} P ; P^{-}$and $\alpha^{n} P^{-}$are below $Q ; \alpha^{n} P^{+}$is on the right of $P$ and so by Lemma $1.2 \alpha^{-n} P^{+}$is on the left of $P$ and so $P^{+}$is on the left of $\alpha^{n} P$ ). So the boundary of $X / \lambda$ is compact, and so $X / \lambda$ is either compact or contains the end of $\tilde{M} / \lambda$, but $X / \lambda$ doesn't contain the end because it doesn't contain $P^{+} / \lambda$.

Lemma 1.5. If $\alpha$ does not stabilize $P$, then no power of $\alpha$ does.

Proof. Apply Lemma 1.4.

Corollary (Casson). The double curves of a least area map $f: T^{2} \rightarrow M$ represent primitive elements of $\pi_{1}\left(T^{2}\right)$.

Lemma 1.6. If $P$ intersects $\alpha P$, then $P$ intersects $\alpha^{n} P$ for all $n$.

Proof. We know that $P$ intersects $\alpha P$ if and only if it intersects $\alpha^{-1} P$. Assume inductively that $\alpha^{i} P$ intersects $P$. Lemmas 1.2 and 1.4 imply that for $i>0, \alpha^{i} P^{+}$ and $\alpha^{-1} P^{+}$lie on different components of $\tilde{M}-P$. This is all we need in the proof of Lemma 1.3 to show that $\alpha^{i} P$ intersects $\alpha^{-1} P$, and so $\alpha^{i+1} P$ intersects $P$. 


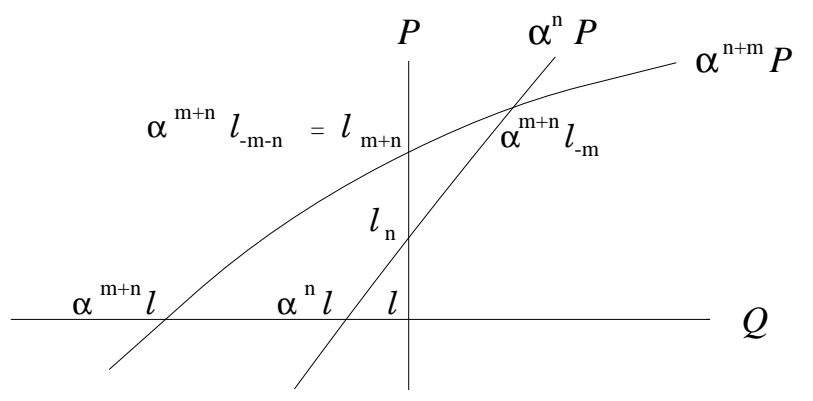

Figure 4

Now to prove the theorem we may assume that all the translates of $P$ by the action of $\alpha$ are different and intersect each other. Observe that only finitely many planes can intersect a tubular neighborhood $N$ of each line (they project to different annuli in $N / \lambda$, which is compact and so its projection into $M$ is finite to 1$)$. In particular, no power of $\alpha$ can stabilize $l$ : if $\alpha^{n} l=l$, then the planes $P, \alpha^{n} P, \alpha^{2 n} P, \ldots$ would all intersect at $l$. Now if 3 planes meet pairwise, then the 3 lines of intersection are either disjoint or they all meet, because $\left(P_{i} \cap P_{j}\right) \cap\left(P_{i} \cap P_{k}\right)=P_{i} \cap P_{j} \cap P_{k}$. Since only finitely many lines intersect a neighborhood of $l, \alpha^{n} l \cap l=\emptyset$ and $l_{n} \cap l=\emptyset$ for some $n$. Thus ..., $\alpha^{-2 n} l, \alpha^{-n} l, l, \alpha^{n} l, \alpha^{2 n} l, \ldots$ are parallel and disjoint, and appear in this order in $Q$, and if $m$ is a positive multiple of $n$, then $l_{m}$ and $l_{n}$ are in the same component of $\tilde{M}-Q$ (otherwise $\alpha^{m} P^{+}$and $\alpha^{n} P^{+}$would have to be on different components of $\tilde{M}-P)$.

Look at the configuration in Figure 4: $l, \alpha^{n} l$ and $\alpha^{m+n} l$ are disjoint parallel lines in $Q$, and $\alpha^{n} l$ lies between $l$ and $\alpha^{m+n} l$. Also, if $m$ is large enough, $l, l_{n}$ and $l_{m+n}$ are disjoint parallel lines in $P$, and $l_{n}$ lies between $l$ and $l_{m+n}$. Thus $\alpha^{m+n} l$, $\alpha^{m+n} l_{-m-n}$ and $\alpha^{m+n} l_{-m}$ are parallel and disjoint in $\alpha^{m+n} P$, and $\alpha^{m+n} l_{-m-n}$ lies between $\alpha^{m+n} l$ and $\alpha^{m+n} l_{-m}$. So in $P, l_{-m-n}$ lies between $l$ and $l_{-m}$. By the same argument $l_{-m-2 n}, l_{-m-3 n}, l_{-m-4 n}, \ldots$ lie between $l$ and $l_{-m}$, and this contradicts the fact that only finitely many $l_{i}$ 's intersect each neighborhood of $l$.

Corollary 1. If $M$ is a closed, orientable and irreducible 3-manifold, then the double curves of any least area incompressible immersion $f: T^{2} \rightarrow M$ are simple in $T^{2}$.

The double curves need not be simple in $M$ : there are least area incompressible immersions $f: T^{2} \rightarrow M$ with $M$ covered by $S^{1} \times S^{1} \times S^{1}$, whose pairs of double curves are not homotopic in $T^{2}$, so they must intersect. Gulliver and Scott [3] found the example in Figures 1b and 1c: 2 homotopic least area tori in a Seifert manifold, one with disjoint double curves and the other with curves that intersect. The example below shows that this can happen with any number of double curves, but still one can say something about the intersections in $M$. A surface $S$ immersed in $M$ is said to have the $k$-plane property if given $k$ planes in the preimage of $S$ in $\tilde{M}$, there are 2 of them which are disjoint. With the same hypothesis of Corollary 1 we have:

Corollary 2. A least area incompressible torus with $n$ double curves in its image has the $2 n+2$ plane property. 

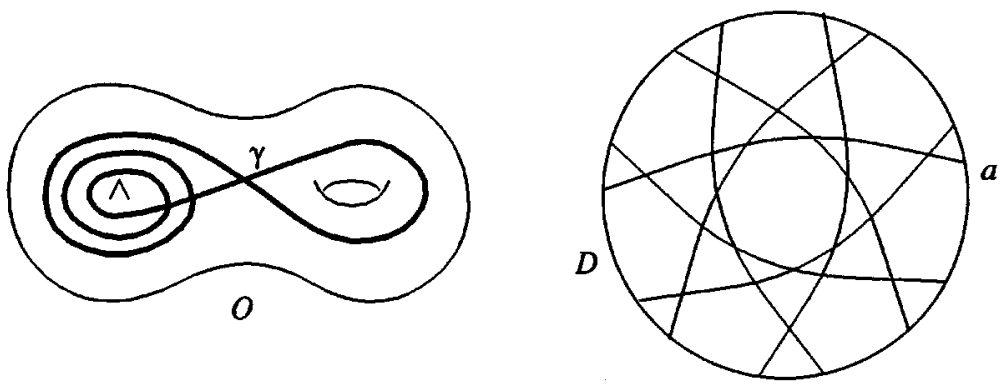

FiguRe 5

Proof. By Theorem 1 the planes which intersect a plane $P$ are divided into $2 n$ families of disjoint planes, so no more than $2 n$ of the planes which intersect $P$ can intersect each other.

Example. Let $M$ be a Seifert fibre space which has an exceptional fibre of order $2 n+1$ and whose base orbifold $O$ has positive genus. Take a vertical torus $T$ in $M$ which projects to a curve $\gamma$ in $O$ which goes $n$ times around the cone point of order $2 n+1$ and once around some essential loop in $O$ (see Figure 5a). By looking at a neighborhood of the exceptional fibre of order $2 n+1$, one can see that there is a disc $D$ in $M$ which is transverse to the fibres and intersects $T$ along $2 n+1 \operatorname{arcs}$ in a star-shaped pattern as in Figure 5b. Thus $T$ has $n$ double curves but it doesn't have the $2 n+1$ plane property, so Corollary 2 is sharp.

Now take an $\operatorname{arc} a$ in $T \cap D$ which divides $D$ into two discs $D^{\prime}$ and $D^{\prime \prime}$. Homotope $T$ in a neighborhood of $a$ (pushing $T$ along $D^{\prime}$ ) to remove that intersection with $D$, and do a similar operation with a disc parallel to $D^{\prime \prime}$. The torus $T^{\prime}$ obtained in this way is homotopic to $T$ and still has the 1-line property, but now all the double curves in $T^{\prime}$ intersect each other, and so every double curve intersects itself and every other double curve in $M$. One can show using the methods of [4] that $T^{\prime}$ is a least area torus for some Riemannian metric in $M$.

In general, the double curves of a least area map from a surface different from the torus into a manifold $M$ are not simple in the surface. If the map has the 1-line property, however (and $M$ is as before), then the double curves cannot represent proper powers of other curves in the surface:

Corollary 3. If $F$ is an orientable surface and $f: F \rightarrow M$ has the 1-line property, then the double curves of $f$ represent (the conjugacy class of) primitive elements of $\pi_{1}(F)$.

Proof. Suppose that a double curve in $F$ is freely homotopic to $\alpha^{n}$ for some $\alpha \in$ $\pi_{1}(F), n>1$. Let $Q$ be one of the planes in the preimage of $f(F)$ in $\tilde{M}$. Identify $\pi_{1}(M)$ with the group of covering translations of $\tilde{M}$, and $\pi_{1}(F)$ with a subgroup $S_{Q}$ which stabilizes $Q$ and acts on it as the group of covering translations of the universal covering of $F$. Each line in the preimage of the double curve in $Q$ is stabilized by a conjugate of $\alpha^{n}$. If $l$ is stabilized by $\alpha^{n}$, then $\alpha l, \alpha^{2} l, \ldots, \alpha^{n} l=l$ are also stabilized by $\alpha^{n}$, and so they are parallel lines in $Q$ and they intersect each other at an infinite number of points. Now if $P$ is the plane that intersects $Q$ at $l$, then $P$ intersects each $\alpha^{i} P$ at a line $l_{i}$, and as $l_{i}$ intersects $l$ at an infinite number 
of points, then $l_{i}$ is also parallel to $l$. Lemma 1.4 applies in this situation, so by Lemma 1.5 no power of $\alpha$ can stabilize $P$.

\section{REFERENCES}

[1] A.Casson, The Torus Theorem, notes from a talk.

[2] M.Freedman, J.Hass, P.Scott, Least area incompressible surfaces in 3-manifolds, Invent. Math. 71 (1983), 609-642. MR 85e:57012

[3] R.Gulliver, P.Scott Least area surfaces can have excess triple points, Topology 26-3 (1987), 345-359. MR 88k:57018

[4] M.Neumann-Coto Least area and minimal intersection of immersed surfaces, in preparation.

Instituto de Matemáticas, UNAM, México D.F. 04510, Mexico

E-mail address: max@math.unam.mx 